\title{
Prion data suggest BSE link to sporadic CJD
}

Declan Butler

Predicting the number of cases of Creutzfeldt-Jakob disease (CJD) in people as a result of transmission of bovine spongiform encephalopathy (BSE) has just got more difficult.

Whereas it was thought that BSE only caused a new form of the disease called variant CJD (vCJD), a study in mice from a team led by John Collinge at University College London suggests that it may also cause a disease indistinguishable from the commonest form of classical, or 'sporadic', CJD (E. A. Asante et al. EMBO J. 21, 6358-6366; 2002). $\Xi$ If the group's mouse model is relevant to the

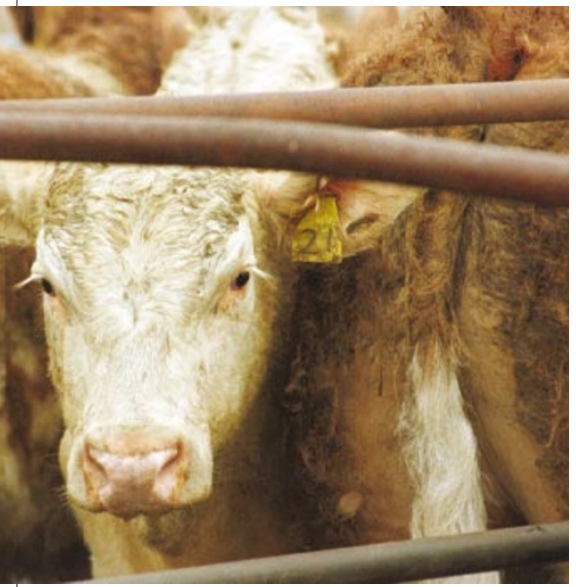

Could transmission of BSE to humans be responsible for more than one type of CJD? human disease, the results also suggest that the true extent of infection may be difficult to assess because of the large number of asymptomatic carriers.

The latest work uses mice engineered to carry the human gene for a cell-membrane protein called PrP. Prion diseases occur when PrP is converted to the abnormal 'prion' form, $\operatorname{PrP}^{\mathrm{Sc}}$. Collinge has developed a test, based on a standard western blot for analysing proteins, to study $\operatorname{PrP}^{\mathrm{Sc}}$ extracted from the brain. This previously showed disease caused by BSE or vCJD to give a characteristic molecular signature that is distinct from sporadic CJD (J. Collinge, K. C. L. Sidle, J. Meads, J. Ironside and A. F. Hill Nature 383, 685-690; 1996).

In their latest experiments, Collinge and his team injected material from the brains of cows with BSE or people with vCJD directly into the brains of two strains of mice with a human PrP genotype known as $129 \mathrm{MM}$. Almost $40 \%$ of the British population has this genotype. In one mouse strain, those that became infected showed the usual BSE pattern in the western blot. But in the other, Collinge's team tested 11 mice infected with BSE material using the western blot. Ten of them showed a pattern consistent with sporadic CJD.

The number of cases of sporadic CJD have been rising in Britain since the 1970s, and this had been attributed to better monitoring for the condition. But in July, researchers led by Adriano Aguzzi of the
University Hospital Zurich reported a sudden increase in sporadic CJD figures in Switzerland in 2001, and suggested that infection with BSE might be to blame (see Nature $418,266 ; 2002)$. Collinge's new data provide worrying molecular evidence that BSE might be to blame for the rise in sporadic CJD.

In previous experiments, Collinge had injected BSE and vCJD material into mice with another human PrP genotype, known as $129 \mathrm{VV}$. The new data are thought to be more relevant to the transmission of BSE because all of the known human victims of VCJD have the 129MM genotype. Another worrying finding is that the $129 \mathrm{MM}$ mice seem to be more susceptible to developing a subclinical infection, with no obvious symptoms.

If a large pool of the British population is carrying a subclinical BSE infection, this would have serious consequences for the potential transmission of the disease, for instance through contaminated surgical instruments. And although laboratory mice are short-lived, infected humans might go on to develop the disease later in life.

The UK Department of Health, which has been briefed by Collinge on his findings, says that it will ask its Spongiform Encephalopathy Advisory Committee to consider the results closely at its next meeting in February. Collinge says that an urgent nationwide screening of tonsil material is needed to get a better estimate of the level of infection in the population.

\section{Europe urged to provide boost for bioterror research}

\section{Alison Abbott, Berlin}

Bioterrorism may be the word on everybody's lips at the moment, but Europe needs to put its money where its mouth is, researchers at the continent's first scientific meeting on the topic were told last week.

A lack of investment in bioweapons research could leave Europe vulnerable to attack, speakers at the meeting asserted.

Under the European Union's Sixth Framework Programme for research funding, "virtually no money is specifically earmarked for such research", said Stefan Kaufmann, a director of the Max Planck Institute for Infection Biology in Berlin. By contrast, the US National Institutes of Health has earmarked more than $\$ 1.5$ billion for a very broad programme, he added.

"If there were a bioterrorist incident in Europe there would be uproar," Kaufmann warned the meeting, "so politicians need to take the issue of research seriously - and now."

The meeting, a symposium on bioterror and bioweapons held in Berlin on 30 November-1 December, came as Britain revealed that it plans to stockpile about 60 million doses of smallpox vaccine in preparation for a bioterrorist attack.

The sixth Framework, which will issue its first call for applications later this month, supports research into infectious diseases, but focuses on the three conditions linked to poverty - tuberculosis, malaria and AIDS - rather than those with weapons potential, such as anthrax and Ebola. Scientists can apply for funds to study the latter, but only under narrow programmes such as 'rational development of antiviral drugs' or 'combating resistance to antibiotics'.

But researchers at the meeting questioned whether the huge infusion of funds into US bioterrorism research will be

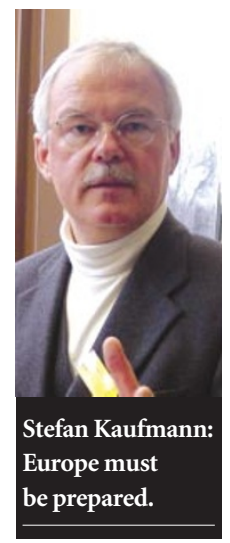

used effectively, and said that it should not necessarily be matched in Europe. Critics have also expressed fears that the expansion of such research in the United States will heighten the risk of bioterrorism, by spreading materials and specialist knowledge.

Milton Leitenberg, an arms-control expert from the University of Maryland, College Park, questioned the fundamental philosophy of large programmes specifically targeting bioterrorism. "There would be a greater spin-off for military purposes by putting money into diseases that are the biggest public-health threat, such as HIV and tuberculosis," he said. 